\title{
MEDIDA DE LA INDEPENDENCIA FUNCIONAL CON ESCALA FIM EN LOS PACIENTES CON EVENTO CEREBRO VASCULAR DEL HOSPITAL MILITAR CENTRAL DE BOGOTÁ EN EL PERIODO OCTUBRE 2010 - MAYO 2011
}

\author{
Andersson L Rozo M.D. ${ }^{1}$ Alberto Jiménez Juliao. M.D. ${ }^{2}$ \\ ${ }^{1}$ Especialista en Medicina Física y Rehabilitación, Universidad Militar Nueva Granada, \\ ${ }^{2}$ Especialista en Medicina Física y Rehabilitación, Universidad Militar Nueva Granada y Hospital Militar Central. \\ Correspondencia: anderssonrozo@hotmail.com
}

Recibido: Julio 22 de 2013 Aceptado: Octubre 10 de 2013

\begin{abstract}
Resumen
El evento cerebro vascular (ECV) es la enfermedad neurológica más prevalente en el adulto. Provoca alto índice de discapacidad en nuestro medio. Con la medición del nivel de discapacidad se puede determinar el impacto que tiene el evento cerebro vascular en el paciente y de acuerdo con éste se fijan las metas en rehabilitación, individualizando las prioridades en la rehabilitación de acuerdo a los hallazgos en la valoración clínica. Objetivo: Medir la dependencia funcional que presentan los pacientes del Hospital Militar Central (HMC), posterior a sufrir un evento cerebro vascular con la escala FIM (Independence Functional Measure). Metodología: De manera retrospectiva, se hizo una revisión de las historias clínicas, midiendo el resultado total y en cada uno de sus ítems evaluados. Se analizaron estos resultados para determinar en qué actividades de la vida diaria presentan mayor discapacidad los pacientes. Resultados: Los pacientes que presentaron evento cerebro vascular en el HMC, presentan afectación significativa de la funcionalidad de acuerdo a los hallazgos al valorar con escala FIM. El compromiso es mayor en el dominio motor que en el cognitivo. Las actividades más afectadas son aquellas que requieren desplazamientos largos o uso de escaleras y las menos comprometidas son la interacción social y la comprensión. Conclusiones: Este estudio mostró que el ECV afecta la funcionalidad del paciente y por lo tanto su calidad de vida, esto hace importante la evaluación objetiva de la funcionalidad, ya que permite establecer metas individuales en rehabilitación, por lo cual las escalas de valoración funcional deben realizarse siempre para hacer objetiva esta medición.
\end{abstract}

Palabras clave: Evento cerebro vascular, escala FIM, funcionalidad.

\section{FUNCTIONAL INDEPENDENCE MEASURE WITH SCALE FIM IN PATIENTS WITH VASCULAR BRAIN EVENTS OF CENTRAL MILITARY HOSPITAL FROM BOGOTA IN THE PERIOD OCTOBER 2010 - MAY 2011}

\begin{abstract}
The cerebrovascular event I the most neurological prevalent disease on adults, causes a great amount of disability on this medium, can be classified by his cause as ischemic or hemorrhagic, by measuring the level of disability can determine the impact of the cerebrovascular event In our patients an accordingly fixing rehabilitation goals, identifying goals, identifying priorities that are required according to the findings of the clinics assessment. Objectives: Measuring the functional dependency presenting on
\end{abstract}


patients of central military hospital after suffering cerebrovascular event with the FIM (functional independence measure) scale. Methodology: In retrospect it was made a chart review, measuring the total score an each of the items evaluated and analyzed these results to determine which of the life activities show greater disability on patients. Results: Patients presented in military hospital with cerebrovascular event is affected in significant functionality according to the findings of the assess whit the FMI scale the commitment is higher in the motor domain than the cognitive domain the most affected activities are those requiring long displacements or the use of ladders an less are committed to social interaction an understanding. Conclusions: This study showed that cerebrovascular event affect the functionality of the patient and thus their quality of life, this makes it important to the objective evaluation of the functionality and allows you to set individual goals of rehabilitation whereby scales functional assessment must be always performed objectively to measurement.

Keywords: Cerebrovascular event, FIM scale, functionality.

\title{
MEDIDA DA INDEPENDÊNCIA FUNCIONAL COM ESCALA FIM NOS PACIENTES COM EVENTO CÉREBRO VASCULAR DO HOSPITAL MILITAR CENTRAL DE BOGOTÁ NO PERÍODO OUTUBRO 2010 - MAIO 2011
}

\begin{abstract}
Resumo
O evento cérebro vascular (ECV) é a doença neurológica mais prevalente no adulto. Provoca alto índice de discapacidade em nossomeio. Com a medição do nível de discapacidade pode-se determinar o impacto que tem o evento cérebro vascular no paciente ede acordo a este se fixam as metas em reabilitação, individualizando as prioridades na reabilitação de acordoàs descobertas na valoração clínica. Objetivo: Medir a dependência funcional que apresentam os pacientes do Hospital Militar Central (HMC) posterior a sofrer um evento cérebro vascular com a escala FIM (Independence Functional Measure). Metodologia: De maneira retrospectiva, se fez uma revisão das historias clínicas, medindo o resultado total e em cada um de seusitens avaliados. Analisaram-seestes resultados para determinar em queatividades da vida diáriaapresentam maior discapacidade os pacientes. Resultados: Os pacientes que apresentaram evento cérebro vascular no HMC apresentam afetação significativa da funcionalidade de acordo às descobertas ao valorar com escala FIM. O compromisso émaior no domínio motor do que no cognitivo. As atividades mais afetadas são aquelas que requerem deslocamentos longos ou uso de escadas $e$ as menos comprometidas são a interação social $e$ a compreensão. Conclusões: Este estudo mostrou que o ECV afeta a funcionalidade do paciente e pelo tanto suaqualidade de vida, istofaz importante aavaliação objetiva da funcionalidade já que permite estabelecer metas individuais em reabilitação pelo qual as escalas de valoração funcional devem realizar-se sempre para fazer objetiva esta medição.
\end{abstract}

Palavras chave: Evento cérebro vascular, Escala FIM, Funcionalidade. 


\section{Introducción}

El evento cerebro vascular (ECV) es importante porque se trata de una causa común de muerte, discapacidad, dependencia y estancia hospitalaria en nuestro medio.

La organización Mundial de la Salud lo define como: "Un síndrome clínico caracterizado por el rápido desarrollo de signos de alteración focal o global de la función cerebral, que dura más de 24 horas o que conducen a la muerte, sin otra causa aparente que la de origen vascular" (1).

La definición de ECV se basa en el mecanismo causal de lesión focal y el área anatómica que se encuentra afectada. Por categorías se ha definido de tipo arterial con infarto secundario a aterosclerosis, embolia de origen cardiaco, enfermedad de pequeños vasos sanguíneos, favorecido por estados de hipercoagulabilidad, enfermedad de células falciformes y los infartos de causa indeterminada (2).

La Organización Mundial de la Salud (OMS) y la Clasificación internacional de Funcionalidad (CIF), ofrecen el siguiente marco para considerar el impacto del ECV en el individuo (3):

\begin{tabular}{|l|l|}
\hline \multicolumn{1}{|c|}{ Concepto } & \multicolumn{1}{c|}{ Definición } \\
\hline $\begin{array}{l}\text { Patología (Enfermedad o } \\
\text { Diagnóstico) }\end{array}$ & Nivel de órgano o sistema. \\
\hline $\begin{array}{l}\text { Deficiencia (Signos y } \\
\text { síntomas) }\end{array}$ & $\begin{array}{l}\text { Manifestaciones de todo el } \\
\text { cuerpo. }\end{array}$ \\
\hline $\begin{array}{l}\text { Limitación para la } \\
\text { actividad (Discapacidad) }\end{array}$ & Alteración de la función. \\
\hline $\begin{array}{l}\text { Restricción de la } \\
\text { participación (Minusvalía) }\end{array}$ & $\begin{array}{l}\text { Situación de inferioridad en } \\
\text { que se encuentra una persona } \\
\text { con discapacidad en la posición } \\
\text { social y roles. }\end{array}$ \\
\hline
\end{tabular}

De acuerdo con lo anterior, la patología es el diagnóstico de evento cerebro vascular. Deficiencia es la lesión isquémica-necrótica que origina el cuadro de déficit neurológico. Las limitaciones para la actividad son las funciones que fueron alteradas debido a la deficiencia presentada y las restricciones para la participación son las repercusiones sociales, laborales, familiares, lúdicas, etc. que se presentan secundarias a las discapacidades generadas por la enfermedad.

La especialidad de Medicina Física y Rehabilitación, por definición, es la responsable del diagnóstico, evaluación, prevención y el tratamiento de la discapacidad. Todos estos procedimientos encaminados a facilitar, mantener o devolver el mayor grado de capacidad funcional e independencia posible al paciente.

Su papel en el ECV se puede orientar hacia tres fines:

1. Valorar las lesiones y el déficit funcional en un momento dado y su evolución.

2. Hacer una estimación del pronóstico más probable-

3. Teniendo en cuenta lo anterior, establecer un plan terapéutico individualizado para cada paciente (4).

Es responsabilidad del médico especialista en Medicina Física y Rehabilitación realizar la historia clínica orientada a la detección de factores de riesgo, examen físico completo enfocado en describir las alteraciones neurológicas presentes y la aplicación de escalas funcionales que permitan elaborar el diagnóstico, definir prioridades, determinar el pronóstico y de acuerdo con todo lo anterior fijar metas y objetivos individuales. Además de dirigir y orientar el equipo de rehabilitación para que sean cumplidas todas las actividades programadas en el plan de rehabilitación.

Existen una serie de pruebas y escalas específicas que permiten registrar de manera objetiva la situación del paciente en cada momento facilitando la observación de cambios evolutivos. En Rehabilitación, las escalas de valoración funcional constituyen una de las principales herramientas para el diagnóstico. Estas escalas permiten evaluar el impacto del evento cerebro vascular en la vida del paciente, algunas de ella permiten calificar el grado en que se encuentran las deficiencias presentadas en el momento de la valoración, otras van a permitir evaluar el grado de discapacidad y dependencia funcional en que se encuentra el paciente, otras determinaran el grado de desventaja en que se encuentra el paciente secundario a la patología.

Para evaluar la deficiencia se encuentran: NIHSS (Impacto agudo del ECV), Ahsworth modificada (Espasticidad), Daniels modificada (Fuerza) (11).

Para evaluar la discapacidad y el grado de dependencia del paciente: FIM (Independence Functional Measure), Barthel, Rankin Modificada, Fulg Meyer (Funcionalidad de miembro superior), HADS (Ansiedad y Depresión) (11). Para evaluar la restricción para la participación: 
índice de actividades instrumentales de Lawton y Brody. Para evaluar la calidad de vida: SF 36.

En la valoración de Medicina Física y Rehabilitación se deben incluir la mayor cantidad de escalas posibles para hacer mas objetivo el resultado. Estas escalas de valoración funcional deben ser válidas, (miden realmente aquello para lo que están destinados), reproducibles o capaces de obtener el mismo resultado en mediciones repetidas en ausencia de variabilidad clínica y, ser sensibles a los cambios clínicos. Es conveniente que además sean prácticos, sencillos y estandarizados, con un método claro de administración y puntuación.

\section{Escala FIM. (Function al Independence Measure)}

La FIM (Functional Independence Measure) es una herramienta mundialmente aceptada como medida de discapacidad, que se ha convertido en el instrumento más ampliamente utilizado para la evaluación del estado funcional de pacientes afectados por trastornos neurológicos. Posee validez para monitorizar los avances en el estado funcional de los pacientes sometidos a tratamiento de rehabilitación. La escala evalúa o se enfoca en áreas prioritarias como el aspecto motor y cognitivo con el fin de planificar la rehabilitación, a su vez permite reconocer y comparar la eficiencia y eficacia, de un tratamiento.

Valora 18 actividades que se agrupan en 2 dimensiones, 13 ítems motores y 5 ítems cognitivos. Estos 18 ítems se agrupan en 6 bloques que evalúan: cuidados personales, control de esfínteres, transferencias, locomoción, comunicación y conciencia del mundo exterior. Incluye 7 niveles que van desde la dependencia completa hasta la independencia. La puntuación final puede oscilar entre 18 puntos (dependencia total) a 126 puntos (independencia completa). Las áreas en las cuales se basa la FIM, permiten valorar el aspecto motor, visto desde: la parte motora como la alimentación, el cuidado personal, el baño, vitales para el desarrollo funcional en las actividades diarias.

Tanto el vestido superior como el vestido inferior, indican las habilidades que puedan tener las diferentes extremidades, y establecer el segmento en que se enfocará el tratamiento. El aseo perineal, valorará que tan minucioso o cuidadoso sea el paciente respecto a su aseo. El control de esfínter tanto vesical como intestinal, refleja los cuidados que debe tenerse con el paciente a la hora de realizar el tratamiento. Las transferencias son importantes ya que en ocasiones es necesario o básico encontrarse en algún lugar como el baño y satisfacer sus necesidades fisiológicas inmediatas, o tan sencillo como la transferencia de la cama a la silla o tan cotidiano como la transferencia del baño a la ducha. La escala también evalúa la deambulación, que permite ver si el paciente tiene alguna deficiencia al movilizarse en la actividad de la marcha o en el manejo de la silla de ruedas, también al subir o bajar escaleras. Debe tenerse en cuenta que estas actividades motoras también requieren el aspecto cognitivo como base para su ejecución, como por ejemplo, la memoria. Por otra parte, el FIM cognitivo que evalúa la comprensión, la expresión, la interacción social, la resolución de problemas y la memoria, permite identificar los factores que el paciente tiene a su favor tanto para una rehabilitación como para su desempeño autónomo en el hogar.

Independencia: No precisa ayuda de otra persona.

7: Independencia completa, todas las habilidades requeridas, es capaz de realizarlas, con seguridad, sin modificación en la conducta, sin ayuda técnica $y$ en un tiempo adecuado.

6: Independencia modificada, la actividad requiere una ayuda técnica, requiere excesivo tiempo o existe algún peligro al realizarla.

Dependencia: El sujeto requiere a otra persona para supervisión o asistencia para realizar la actividad. Requiere a una persona para el cuidado.

5: Supervisión para la realización o dando órdenes sin contacto físico, la ayuda da o coloca las ayudas técnicas.

4: Ayuda con contacto físico mínimo, el sujeto realiza al menos el $75 \%$ del esfuerzo.

3: Asistencia media, el sujeto realiza entre el 50 y el $75 \%$ del esfuerzo.

Dependencia completa: El sujeto realiza menos del $50 \%$ del esfuerzo, máxima o completa ayuda es requerida o la actividad no sería realizada.

2: Máxima asistencia, el sujeto realiza entre el 25 y el $50 \%$ del esfuerzo.

1: Ayuda total, realiza menos del $25 \%$ del esfuerzo. 
Debido a que la escala FIM es práctica, se realiza de rutina en las valoraciones del servicio de Medicina Física y Rehabilitación, es la que se prefiere sobre otras que también aportan información sobre el estado funcional del paciente. La escala Barthel proporciona información valiosa también midiendo la capacidad de realizar actividades de la vida cotidiana en valores de 0 a 100 siendo en este sentido dependencia total a independencia total.

\section{Materiales y métodos}

Estudio descriptivo, retrospectivo. Incluyó pacientes hospitalizados en el HMC, durante el periodo comprendido de octubre del 2010 hasta mayo del 2011 y atendidos por el servicio de Medicina Física y Rehabilitación (MFR).

\section{Criterios de inclusión}

- Paciente con diagnóstico de evento cerebro vascular, que fueron valorados por el Servicio de MFR en los primeros 8 días de la hospitalización.

- Pacientes con primer ictus o recidivante que antes del actual evento eran funcionalmente independientes.

\section{Criterios de exclusión}

- Pacientes valorados después de los 8 días de estar hospitalizados.

- Pacientes a quienes no se les realizo escala FIM en la valoración de MFR.

- Se buscaron en los registros del HMC de Bogotá los pacientes que estuvieron hospitalizados en el periodo octubre 2010 a mayo 2011 con diagnóstico de ECV, Accidente cerebro vascular, Hemorragia Cerebral primaria, ictus.

- Se hizo una revisión de historia clínica verificando que tuvieran la respectiva valoración por MFR.

- Se revisó que tuvieran la escala FIM antes de los 8 días de hospitalización y que hubiera sido diligenciada de manera completa, ordenada y coherente.

- Se realizó el análisis estadístico de los datos obtenidos.

\section{Resultados}

Se encontraron en total 55 historias clínicas de pacientes que habían sido hospitalizados entre octubre de 2010 y mayo de 2001.
De las cuales fueron excluidas 5 historias clínicas: 3 porque la valoración de MFR se realizó luego de los 8 días de hospitalización, y 2 porque no se encontró el resultado de la escala FIM.

\section{Edad}

El promedio de edad para los pacientes fue de 73,3 años, moda de 68 años, con un mínimo de 35 y un máximo de 94 años, con desviación estándar de 12,3.

Gráfico 1. Sexo de los pacientes.

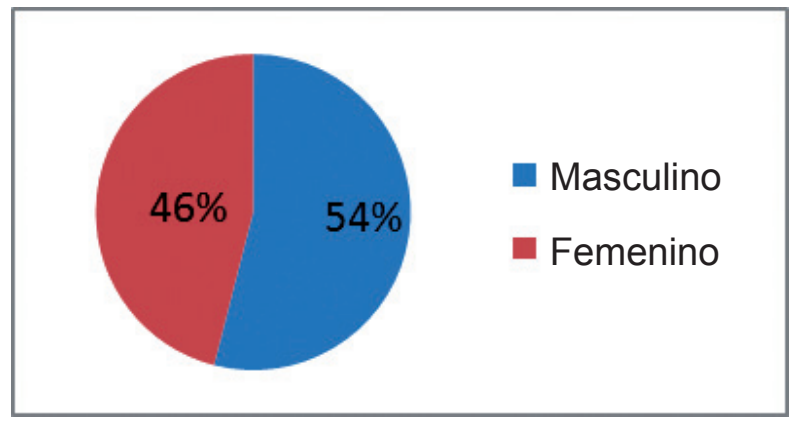

Gráfico 2. Procedencia

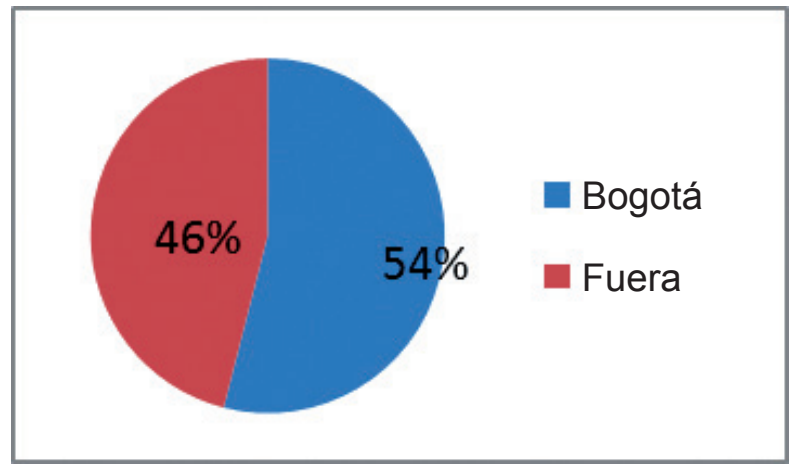

Gráfico 3. Subsistema de salud.

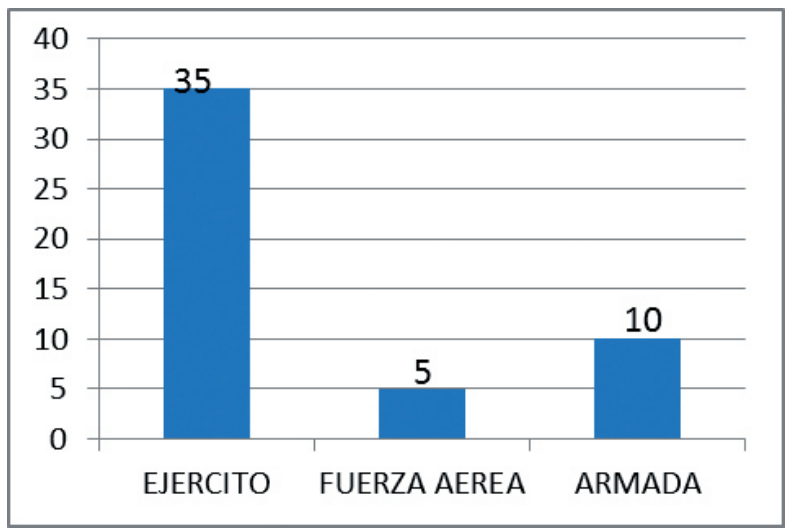


Grafico 4. Tipo de evento.

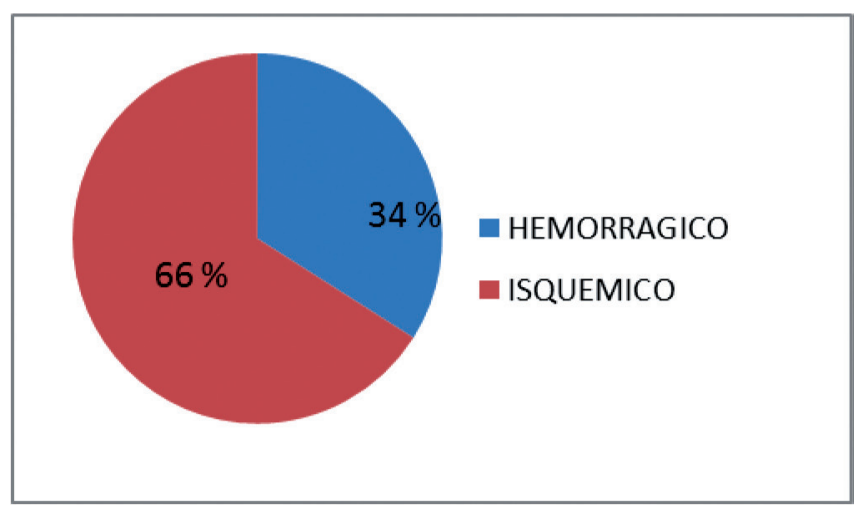

Gráfico 5. Fallecimientos durante la hospitalización.

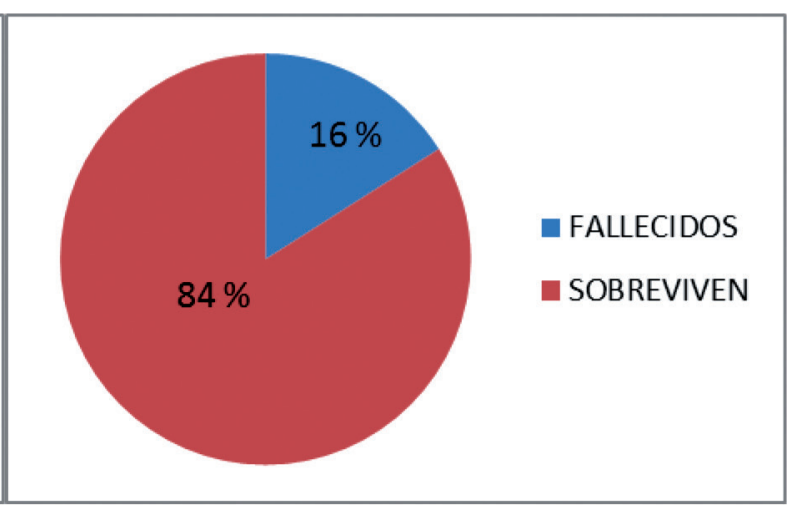

\section{Caracterización de los ítems y sus resultados}

Se cuenta el número de pacientes en cada nivel de independencia con respecto a cada ítem evaluado para determinar cuál es el más frecuente y el menos frecuente.

\begin{tabular}{|c|c|c|c|c|c|c|c|}
\hline ITEM & $\begin{array}{l}\text { ASISTENCIA } \\
\text { TOTAL }\end{array}$ & $\begin{array}{l}\text { ASISTENCIA } \\
\text { MÁXIMA }\end{array}$ & $\begin{array}{l}\text { ASISTENCIA } \\
\text { MODERADA }\end{array}$ & $\begin{array}{l}\text { ASISTENCIA } \\
\text { MÍNIMA }\end{array}$ & SUPERVISIÓN & $\begin{array}{l}\text { INDEPENDENCIA } \\
\text { MODIFICADA }\end{array}$ & $\begin{array}{l}\text { INDEPENDENCIA } \\
\text { COMPLETA }\end{array}$ \\
\hline $\begin{array}{l}\text { ARREGLO } \\
\text { PERSONAL }\end{array}$ & 9 & 6 & 4 & 5 & 6 & 12 & 8 \\
\hline ALIMENTACIÓN & 5 & 6 & 7 & 10 & 11 & 8 & 3 \\
\hline BAÑO & 7 & 9 & 9 & 12 & 7 & 4 & 2 \\
\hline VESTIDO SUP. & 7 & 9 & 9 & 12 & 7 & 4 & 2 \\
\hline VESTIDO INF. & 8 & 9 & 10 & 10 & 8 & 3 & 2 \\
\hline ASEO PERINEAL & 10 & 4 & 8 & 12 & 8 & 5 & 3 \\
\hline VEJIGA & 14 & 1 & 4 & 8 & 10 & 7 & 6 \\
\hline INTESTINO & 15 & 1 & 4 & 7 & 9 & 7 & 7 \\
\hline CAMA - SILLA & 11 & 5 & 7 & 5 & 10 & 7 & 5 \\
\hline EN BAÑO & 9 & 6 & 6 & 10 & 8 & 7 & 4 \\
\hline EN DUCHA & 12 & 3 & 6 & 12 & 10 & 6 & 1 \\
\hline DEAMBULACIÓN & 11 & 8 & 5 & 8 & 9 & 8 & 1 \\
\hline ESCALERAS & 14 & 6 & 6 & 11 & 7 & 5 & 1 \\
\hline COMPRENSIÓN & 4 & 2 & 9 & 12 & 7 & 7 & 9 \\
\hline EXPRESIÓN & 4 & 7 & 5 & 7 & 12 & 7 & 8 \\
\hline INTERACCIÓN & 3 & 2 & 12 & 7 & 11 & 6 & 9 \\
\hline PROBLEMAS & 5 & 7 & 9 & 10 & 10 & 6 & 3 \\
\hline MEMORIA & 6 & 2 & 8 & 7 & 10 & 10 & 7 \\
\hline TOTAL & 152 & 90 & 126 & 163 & 164 & 123 & 82 \\
\hline
\end{tabular}




\section{FIM total}

Para describir los valores de FIM total se realizó una discriminación por percentiles y cuartiles, ubicando los de mayor relevancia.

\begin{tabular}{|c|c|}
\hline & Percentiles \\
\hline $1,0 \%$ & 18,0 \\
\hline $5,0 \%$ & 20,0 \\
\hline $10,0 \%$ & 29,5 \\
\hline $25,0 \%$ & 44,0 \\
\hline $50,0 \%$ & 74,0 \\
\hline $75,0 \%$ & 91,0 \\
\hline $90,0 \%$ & 104,5 \\
\hline $95,0 \%$ & 111,0 \\
\hline $99,0 \%$ & 126,0 \\
\hline
\end{tabular}

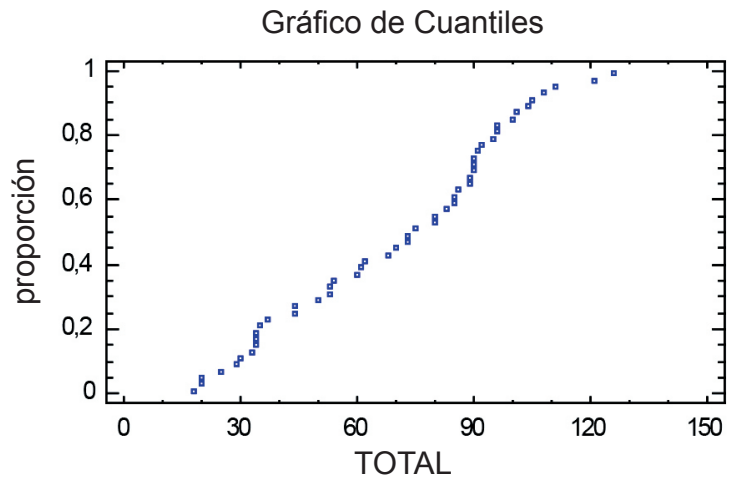

- El $75 \%$ de los pacientes evaluados tiene 91 puntos o menos con respecto al valor total y el otro $25 \%$ tiene 91 puntos o más.

- El $50 \%$ de los pacientes evaluados tiene 74 puntos o menos con respecto al valor total y el otro $50 \%$

tiene 74 puntos o más. Siendo este valor la misma mediana.

- $\quad$ El $25 \%$ de los pacientes evaluados tiene 44 puntos o menos con respecto al valor total y el otro $75 \%$ tiene 44 puntos o más.

\section{FIM Dominio motor}

Para describir los valores de FIM motor se realizó una discriminación por percentiles y cuartiles, ubicando los de mayor relevancia.

\begin{tabular}{|c|c|}
\hline & Percentiles \\
\hline $1,0 \%$ & 13,0 \\
\hline $5,0 \%$ & 13,0 \\
\hline $10,0 \%$ & 15,5 \\
\hline $25,0 \%$ & 28,0 \\
\hline $50,0 \%$ & 52,5 \\
\hline $75,0 \%$ & 65,0 \\
\hline $90,0 \%$ & 75,0 \\
\hline $95,0 \%$ & 78,0 \\
\hline $99,0 \%$ & 91,0 \\
\hline
\end{tabular}

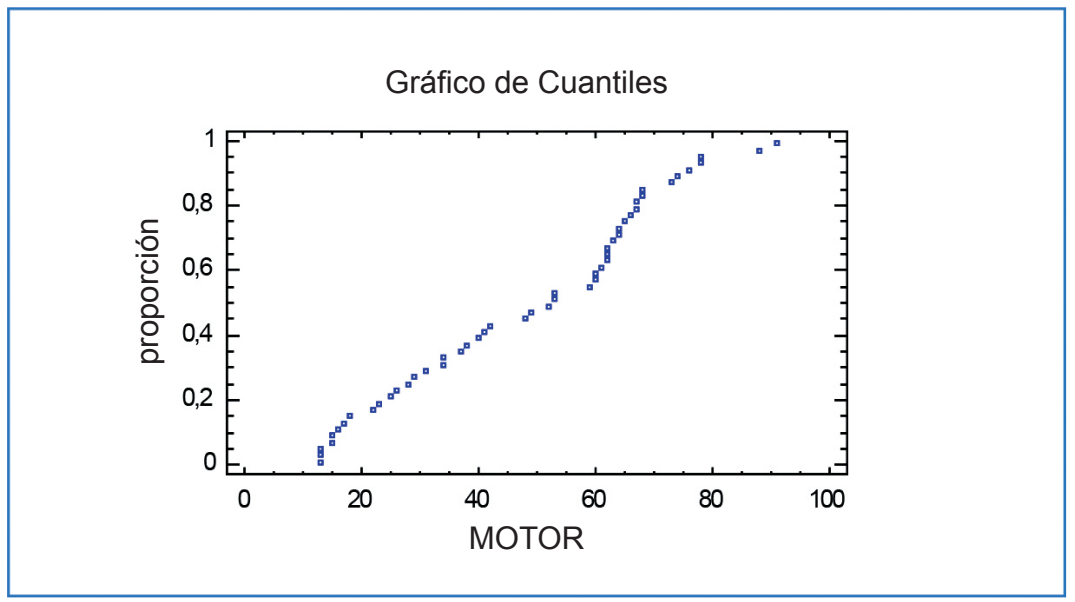

- $\mathrm{El} 75 \%$ de los pacientes evaluados tiene 65 puntos o menos con respecto al valor total y el otro $25 \%$ tiene 65 puntos o más.

- El $50 \%$ de los pacientes evaluados tiene 52,5 puntos o menos con respecto al valor total y el

otro $50 \%$ tiene 52,5 puntos o más. Siendo este valor la misma mediana.

- $\quad$ El $25 \%$ de los pacientes evaluados tiene 28 puntos o menos con respecto al valor total y el otro $75 \%$ tiene 28 puntos o más. 


\section{FIM Dominio cognitivo}

Para describir los valores de FIM cognitivo se realizó una discriminación por percentiles y cuartiles, ubicando los de mayor relevancia.

\begin{tabular}{|c|c|}
\hline & Percentiles \\
\hline $1,0 \%$ & 5,0 \\
\hline $5,0 \%$ & 5,0 \\
\hline $10,0 \%$ & 11,0 \\
\hline $25,0 \%$ & 16,0 \\
\hline $50,0 \%$ & 22,0 \\
\hline $75,0 \%$ & 28,0 \\
\hline $90,0 \%$ & 32,5 \\
\hline $95,0 \%$ & 33,0 \\
\hline $99,0 \%$ & 35,0 \\
\hline
\end{tabular}

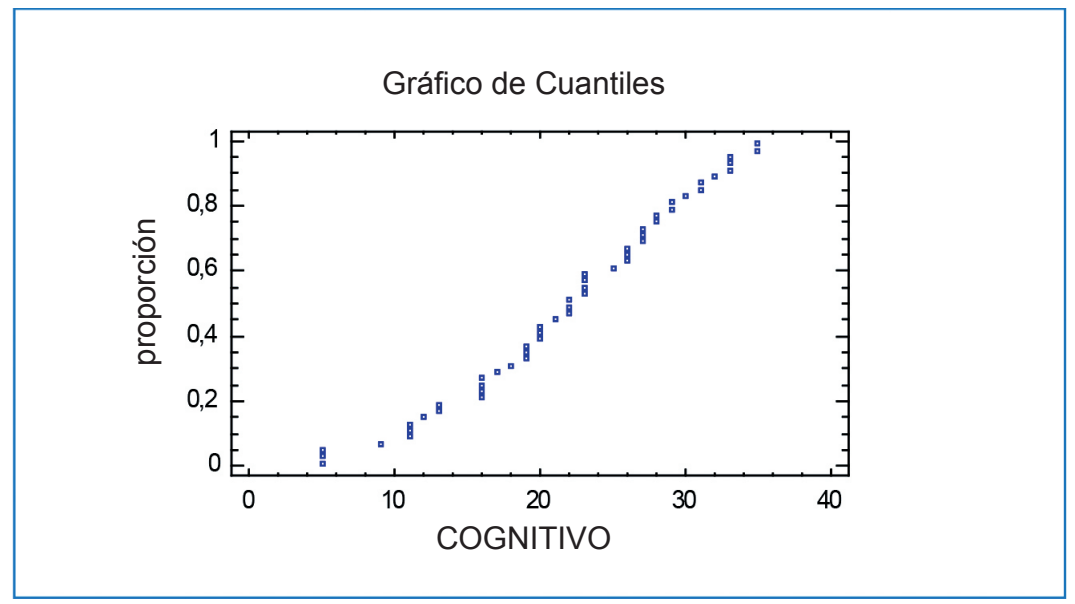

- El $75 \%$ de los pacientes evaluados tiene 28 puntos o menos con respecto al valor total y el otro $25 \%$ tiene 28 puntos o más.

- El $50 \%$ de los pacientes evaluados tiene 22 puntos o menos con respecto al valor total y el otro $50 \%$ tiene 22 puntos o más. Siendo este valor la misma mediana.

- El $25 \%$ de los pacientes evaluados tiene 16 puntos o menos con respecto al valor total y el otro $75 \%$ tiene 16 puntos o más.

\section{Discusión}

El análisis del impacto que genera el ECV en nuestra población utilizando la escala FIM nos permite conocer cuáles son aquellas áreas (actividades) en las que se presenta mayor o menor grado de dependencia funcional y de acuerdo con esto fijar las metas y objetivos de rehabilitación (5). Nunca antes en nuestra población se había realizado un análisis de este tipo, a pesar de que dentro de la valoración realizada por parte de la especialidad de Medicina Física y Rehabilitación para los pacientes hospitalizados por evento cerebro vascular está incluida la realización de la escala FIM.

En el año 2001 Paognelli G, Carlo y colaboradores (1), en Chile encontraron que el mayor grado de dependencia funcional estaba en el dominio motor: categorías movilidad y ambulación, con un nivel de asistencia máxima. Dentro del dominio cognitivo las mayores alteraciones se presentaban en la categoría conocimiento social actividades de Memoria, con un nivel de asistencia mínima.
Luego en el año 2004 Counsell Cy colaboradores (25), presentan un modelo de pronóstico para la rehabilitación en evento cerebro vascular donde evidencian que basados en la escala FIM, la mayor dependencia funcional se presenta en el dominio motor, categoría autocuidados, principalmente en las actividades que conciernen a vestido cuerpo superior, vestido cuerpo inferior y aseo perineal con un nivel de asistencia máxima. Dentro del dominio cognitivo las mayores alteraciones se presentaban en la categoría conocimiento social actividades de memoria, con un nivel de asistencia mínima.

En el presente trabajo se realizó el análisis del valor total del FIM en el cual hay un rango normal entre $18 y$ 126, encontrando según la medición por cuartiles que el $75 \%$ de los pacientes evaluados tiene 91 puntos o menos con respecto al valor total y el otro $25 \%$ tiene 91 puntos o más, al mirar el percentil 50 se encontró que el $50 \%$ de los pacientes evaluados tiene 74 puntos o menos con respecto al valor total y el otro $50 \%$ tiene 74 puntos o más, este valor es la misma mediana. Y 
el $25 \%$ de los pacientes evaluados tiene 44 puntos o menos con respecto al valor total y el otro $75 \%$ tiene 44 puntos o más. Lo que deja en evidencia el gran impacto sobre el estado funcional general de los pacientes que se presenta posterior al ictus.

En el análisis del FIM Motor se encontró que el 75\% de los pacientes evaluados tiene 65 puntos o menos con respecto al valor total y el otro $25 \%$ tiene 65 puntos o más. Además, el $50 \%$ de los pacientes evaluados tiene 52,5 puntos o menos con respecto al valor total y el otro $50 \%$ tiene 52,5 puntos o más, este valor es la misma mediana. El $25 \%$ de los pacientes evaluados tiene 28 puntos o menos con respecto al valor total y el otro $75 \%$ tiene 28 puntos o más. Al evaluar el dominio motor se encontró que el ítem que se encuentra más afectado fue vestido superior el cual pertenece a la categoría autocuidado donde la mayoría de los pacientes presentan dependencia entre mínima y moderada. Se atribuye a que, debido al déficit motor presentado y a la alteración que puede tener el tanto en dominio de la mano, como en el equilibrio para poder mantener una postura mientras se coloca el vestido inferior es que este es el que se encuentra más comprometido (25). También se encontró que el ítem menos afectado fue el que corresponde a arreglo personal, es decir: cepillado de dientes, peinarse, lavarse la cara entre otras. Lo cual es positivo, le ofrece independencia al paciente en algunas actividades básicas cotidianas y permitirá un mejor proceso de rehabilitación.

Llama la atención que los resultados en cuanto al control de esfínteres fueron muy similares y la mayoría presentaban dependencia total. Lo cual significa que un grupo mayoritario de los pacientes del hospital presentan secuelas que comprometen los esfínteres y relacionando con revisiones anteriores (25) los hallazgos son similares, además es importante mencionar que la incontinencia urinaria es un factor de mal pronóstico funcional en rehabilitación por lo cual se debe priorizar su estudio y la intervención al respecto en manejo conjunto con urología. (15)

En la categoría movilidad los resultados fueron igualmente similares, tanto para silla-cama, ir al baño y al retrete. Esto significa que los traslados cortos que debe realizar el paciente en su ambiente para realizar actividades básicas como es el aseo y baño están similarmente comprometidos con dependencia entre mínima y moderada. Este punto es interesante ya que en el proceso de rehabilitación se debe incluir el manejo por parte de fisioterapia de la marcha terapéutica, es decir en desplazamientos cortos en el hogar en lo posible supervisado y en terapia ocupacional para poder garantizar la correcta realización final de la actividad que debe desarrollar.

En cuanto a la deambulación es evidente que la afectación motora llevará a que la marcha comunitaria se afecte y es por esto por lo que tanto los desplazamientos largos como el subir o bajar escaleras se comprometen seriamente, siendo el valor 1 el que más se repitió, correspondiente a asistencia total. Dentro de los procesos de rehabilitación esta es de las metas más difíciles de recuperar, ya que no solo hay que evocar el patrón motor perdido o disfuncional, sino también entrenar la marcha con uso de ortésis y devolver la seguridad al paciente principalmente en terrenos irregulares o subiendo y bajando escaleras.

En el caso del dominio cognitivo, los valores en general presentan puntajes mayores, lo cual se considera muy bueno puesto que la capacidad de entender, reconocer y manejar su enfermedad por parte del paciente lleva a una mayor adherencia a los tratamientos de rehabilitación y por lo tanto mejores resultados. Se encontró que el $75 \%$ de los pacientes evaluados tiene 28 puntos o menos con respecto al valor total y el otro $25 \%$ tiene 28 puntos o más. Además, El $50 \%$ de los pacientes evaluados tiene 22 puntos o menos con respecto al valor total y el otro $50 \%$ tienen 22 puntos o más, este valor es la misma mediana. Y el $25 \%$ de los pacientes evaluados tiene 16 puntos o menos con respecto al valor total y el otro $75 \%$ tiene 16 puntos o más.

En cuanto a la categoría comunicación, los resultados mostraron que la afectación no es severa y que el nivel de dependencia se encuentra en los valores 4 para comprensión y 5 para expresión, que significa dependencia mínima o supervisión, respectivamente. Dentro del proceso de rehabilitación se ha evidenciado que la presencia de afasia, ya sea sensitiva (comprensión) o motora (expresión), se relaciona igualmente con mal pronóstico funcional a largo plazo (25).

La categoría conocimiento social, en la cual se evalúa la capacidad del paciente para realizar resolución de problemas básicos de la vida y enfrentar situaciones de estrés menor, además de la memoria a corto y largo plazo, mostró resultados favorables ubicando la mayor parte de este grupo en dependencia mínima o en supervisión. Igualmente se considera que un nivel 
cognitivo que le permita al paciente hacer una introspección de su patología y entendimiento del plan de manejo propuesto por el médico rehabilitador, llevará a mejores resultados en cuanto a funcionalidad a largo plazo $(11,19)$.

Con la realización de este trabajo se hace evidente que el compromiso general del paciente posterior al ictus es significativo siendo mayor en el dominio motor en nuestra población. Además, que las actividades que más se comprometen son aquellas que tienen que ver con desplazamientos o subir y bajar escaleras.

El conocimiento y aplicación de escalas de valoración funcional permite, sin duda alguna, conocer a fondo el impacto que la discapacidad ha generado en la vida del paciente, y las respectivas restricciones para la participación que presenta $(15,25)$.

La evaluación descriptiva permite conocer las discapacidades de los pacientes, para que de este modo las metas en rehabilitación sean propuestas de manera individual, buscando dar la mayor mejoría posible de acuerdo con las necesidades encontradas y que así se logre una reintegración rápida dejando las menores secuelas posibles $(26,27)$.

\section{Conclusiones}

- Los pacientes que presentaron evento cerebro vascular en el Hospital Militar Central de Bogotá sufren afectación significativa de la funcionalidad de acuerdo con los hallazgos al valorar con escala FIM.

- El compromiso es mayor en el dominio motor que en el cognitivo.

- Las actividades más afectadas son aquellas que requieren desplazamientos largos o uso de escaleras, y las menos comprometidas son la interacción social y la comprensión.

- La evaluación objetiva de la funcionalidad permite establecer metas individuales en rehabilitación, por lo cual las escalas de valoración funcional deben realizarse siempre para hacer efectiva esta medición.

\section{Conflictos de interés}

Los autores del trabajo indican que no presentan conflictos de interés y que autorizan la publicación del mismo.

\section{Agradecimientos}

Se agradece a los médicos especialistas y médicos residentes del servicio de Medicina Física y Rehabilitación del Hospital Militar Central por su apoyo y orientación académica.

\section{Referencias}

1. Paolinelli C, González P, Doniez ME, Donoso T, Salinas V. Instrumento de evaluación funcional de la discapacidad en rehabilitación.: Estudio de confiabilidad y experiencia clínica con el uso del Functional Independence Measure. Rev Med Chil. 2001; 129(1): 23-31.

2. Procedures for scoring the functional independence measure (FIM). Uniform Data System for Medical Rehabilitation: Guide for the Uniform Data Set for Medical Rehabilitation (Adult FIM), Version 4.0. Buffalo, NY: State University of New York; 1993.

3. Scott E. Kasner, MD,; Robert J. Adams, MD,; Gregory W. Albers, MD; Ruth L. Bush, MD; Susan C. Fagan. Guidelines for the Prevention of Stroke in Patients With Stroke or Transient Ischemic Attack: A Guideline for Healthcare Professionals From the American Heart Association/American Stroke Association, 2011;42;227-276

4. Jimenez A, Massasa G, Esquivia C. Guía de manejo. Rehabilitación de las secuelas de enfermedad cerebro vascular: Bogotá: Servicio Medicina Física Y rehabilitación, Hospital Militar Central; 2010.

5. Management of patients with stroke: Rehabilitation, prevention and management of complications, and discharge planning. A national clinical guideline. Edimburgo: Scottish Intercollegiate Guidelines network; 2010.

6. Merwick A, albers GW, Amarenco P, Arsava EM, Ay H, Calvet $\mathrm{D}$, et al. Addition of brain and carotid imaging to the $\mathrm{ABCD}$ score to identify patients at early risk of stroke after transient ischaemic attack: a multicentre observational study. Lancet Neurol. 2010; 9: 1060-69.

7. Adamson J, Beswick A, Ebrahim S. Is stroke the most common cause of disability? J Stroke Cerebrovasc Dis. 2004; 13(4): 171-7.

8. World Health Organization. International Classification of Functioning, Disability and Health (ICF). Geneva: The World Health Organization; 2001. [acceso 30 de abril 2010]. Disponible en: http://www.who.int/classifications/icf/en/

9. Langhorne P, Pollock A. Stroke Unit Trialists' Collaboration. What are the components of effective stroke unit care? Age Ageing. 2002; 31(5): 365-71.

10. Parker J, Arbour R, March K. Acute stroke: Pathophysiology, diagnosis, and treatment AACN. Clinical Issues. 2005; 16(4): 2005.

11. Guía clínica para el tratamiento del ACV isquémico, European Stroke organizatión 2008.

12. Continuum: Lifelong Learning Neurol. 2010; 14.

13. Kollen B, Kwakkel G, Lindeman E. Time Dependency of Walking Classification in Stroke, Physical Therapy. 2006; 86(5): 618-25.

14. Mintzopoulos D, Khanicheh A, Konstas AA, Astrakas LG, Singhal $\mathrm{AB}$, Moskowitz MA. Functional MRI of Rehabilitation in Chronic 
Stroke Patients Using Novel MR-Compatible Hand Robots. The Open Neuroimaging Journal. 2008; 2: 94-101.

15. Cuadrado AA. Rehabilitación del ACV: evaluación, pronóstico y tratamiento. Galicia Clin. 2009; 70 (3): 25-40

16. Lew HL, Lombard LA, Reddy CC, Moroz A, Edgley SR, Chae J. Stroke and Neurodegenerative Disorders: 3. Poststroke Rehabilitation. PM R. 2009; 1(3): S19-26.

17. Meschia JF. Ischemic Stroke as a Complex Genetic Disorder, Department of Neurology. Semin Neurol. 2006; 26(1): 49-56.

18. JENNIFER JUHL MAJERSIK, M.D. ELAINE J. SKALABRIN, M.D. , Single-Gene Stroke Disorders Department of Neurology, Mayo Clinic College of Medicine, Jacksonville, Florida. 2006

19. Manual Sermef. Madrid: Sociedad Española de Medicina Física Y Rehabilitación; 2006.

20. Normal Cerebral oxygen and glucose Metabolism. Brain, December 2009; 132: 3274 - 3284

21. Poderes WJ, Videen TO, Markham J. Hemostasis and Thrombosis: Basic Principles and Clinical Practice. Filadelfia: J.B. Lipincott Company; 1994. p.1225.
22. Vincent JL. Molecular mechanisms of ischemic neuronal injury Ann Emerg Med. 2000; 36: 483-506.

23. Delgado G, Aymerich N, Herrera M. The intracerebral haemorrhage. Anales San Navarra. 2000; 23(3):

24. Martinez A. Marcadores biológicos en la hemorragia intracerebral espontánea. Neurología. 2007; 22(7): 448-455

25. Selvarajah JR, Smith CJ, Hulme S, Georgiou RF, Vail A, Tyrrell PJ. Prognosis in patients with transient ischaemic attack (TIA) and minor stroke attending TIA services in the North West of England: the NORTHSTAR Study. J Neurol Neurosurg Psychiatry J R Selvarajah, C J Smith, S Hulme, et al. 2008; 79 (1): 38-43.

26. Meijer R. Prognostic factors for ambulation and activities of daily living in the subacute, phase after stroke. A systematic review of the literature. Clin Rehabil. 2003; 17: 119-29.

27. Counsell C, Dennis M, McDowall M. Predicting functional outcome in acute stroke: comparison of a six variable mode with other predictive systems and informal clinical prediction. J Neurol Neurosurg Psychiatry. 2004; 75(3): 401-5. 